\title{
Transplante de córnea em ceratocone: avaliação dos resultados e complicações obtidos por cirurgiões experientes e em treinamento
}

\author{
Corneal transplantation in keratoconus: evaluation of results and complications \\ obtained by skillful and surgeons in training
}

Vera Lucia Degaspare Monte Mascaro ${ }^{1}$ Marinho Jorge Scarpi ${ }^{2}$

Ana Luisa Hofling'-Lima ${ }^{3}$

Luciene Barbosa de Sousa ${ }^{4}$
Trabalho realizado na Universidade Federal de São Paulo - UNIFESP - São Paulo (SP) - Brasil.

${ }^{1}$ Pós-graduanda da Universidade Federal de São Paulo - UNIFESP - São Paulo (SP) - Brasil.

${ }^{2}$ Livre docente, Departamento de Oftalmologia da UNIFESP - São Paulo (SP) - Brasil.

${ }^{3}$ Professora titular, Chefe do Departamento de Oftalmologia da UNIFESP - São Paulo (SP) - Brasil.

${ }^{4}$ Professora afiliada da UNIFESP - São Paulo (SP) Brasil.

Endereço para correspondência: Vera Lucia D. M. Mascaro. Av. Paulista, 807 - Conj. 1024 - São Paulo (SP) CEP 01311-100

Recebido para publicação em 30.09.2005

Última versão recebida em 07.01.2007

Aprovação em 19.03.2007

\section{RESUMO}

Objetivo: Analisar os resultados refrativos e visuais dos transplantes de córnea em pacientes portadores de ceratocone, em dois grupos de cirurgiões. Métodos: Setenta olhos de setenta pacientes com transplante de córnea por ceratocone realizados por médicos experientes (Grupo I) e em treinamento (Grupo II) foram examinados, após a remoção completa da sutura, e os dados de seus prontuários recolhidos. Os pacientes foram avaliados quanto às características demográficas, técnica de cirurgia empregada, complicações pós-operatórias e de seus resultados refracionais e visuais. Resultados: O tempo de pós-operatório médio foi de 7,9 anos, variando de 1,6 a 20 anos. A proporção homens/mulheres foi de 1:1. Cada grupo foi formado por 35 olhos. A idade do doador foi diferente entre os dois grupos. A acuidade visual pré-operatória diferiu entre os dois grupos, mas não houve relação com a acuidade visual na avaliação clínica pósoperatória. A técnica cirúrgica empregada diferiu apenas no número de suturas. Com relação a todos os resultados refrativos, topográficos e de acuidade visual no pós-operatório, não houve diferença estatística entre os grupos estudados. A principal complicação observada foi a reação de rejeição. A rejeição endotelial foi mais freqüente no Grupo II. Apenas três enxertos não foram transparentes, todos pertencentes ao Grupo II. Conclusão: Os resultados visuais, refracionais e complicações após transplante de córnea por ceratocone foram similares quando realizados por cirurgiões experientes e em treinamento.

Descritores: Ceratocone; Transplante de córnea; Complicações pós-operatórias/rejeição do enxerto; Erros de refração/astigmatismo

\section{INTRODUÇÃO}

Ceratocone anterior é uma doença não inflamatória e progressiva, na qual a córnea assume uma forma cônica, cujo ápice com freqüência localizase centralmente ou em uma posição excêntrica inferior, provocada pelo afinamento e protrusão da córnea. O ceratocone é uma das causas mais comuns de astigmatismo irregular ${ }^{(1)}$.

O tratamento do ceratocone depende da gravidade da doença. Nos estágios iniciais, óculos e lentes de contato são as modalidades de tratamento indicadas. Em casos mais avançados, com astigmatismo corneal irregular elevado e opacidades estromais apicais, em que as lentes de contato não mais proporcionam acuidade visual satisfatória ou sequer são toleradas, a terapêutica cirúrgica deve ser indicada ${ }^{(2-3)}$. 
Muitas técnicas cirúrgicas foram descritas para correção do ceratocone. Dentre elas podem ser citadas a ceratoplatia lamelar ${ }^{(4-7)}$, a epiceratoplastia ${ }^{(8-11)}$ e o implante de anel intracorneal $^{(12-15)}$. Entretanto, o mais comum é que os pacientes portadores de ceratocone sejam tratados primariamente com transplante de córnea ${ }^{(16)}$.

O transplante de córnea é a forma de aloenxerto mais largamente praticada em todo o mundo ${ }^{(17)}$. Refinamentos da técnica cirúrgica e o aumento do entendimento da imunobiologia dos enxertos de córnea têm feito da cirurgia de transplante de córnea um dos maiores sucessos da moderna oftalmologia ${ }^{(18)}$. Apesar disso, tornar a córnea transplantada com refração útil pode ser mais difícil do que a deixar transparente.

Assim, é importante determinar se os enxertos ópticos em portadores de ceratocone são de benefício funcional ao paciente. A análise dos resultados dos transplantes de córnea ao longo do tempo poderia ajudar a identificar os fatores de risco para maus resultados e a direcionar as pesquisas para a solução do problema.

\section{OBJETIVO}

Este estudo teve como objetivo avaliar os resultados de transplantes de córnea primários em pacientes portadores de ceratocone e compará-los, considerando-se a experiência dos cirurgiões.

\section{MÉTODOS}

Este é um estudo transversal com avaliação de dados retrospectivos, no qual foram examinados no período de outubro de 2002 a março de 2003, 70 olhos de 70 pacientes com diagnóstico de ceratocone que haviam sido submetidos a transplante penetrante de córnea primário.

Para serem incluídos neste estudo, os pacientes deveriam ser portadores de ceratocone submetidos a transplante de córnea, tendo todas as suturas removidas, com idade maior ou igual a 18 anos e não possuírem doença sistêmica ou outra doença ocular detectadas à anamnese e exame clínico de rotina. Do paciente com enxerto bilateral, apenas os dados de um dos olhos entraram para análise, por sorteio aleatório.

Este estudo foi aprovado pelo Comitê de Ética da UNIFESPEPM e os pacientes consentiram em participar do estudo, após assinatura do termo de consentimento livre e esclarecido.

Para este estudo, dois médicos experientes (VLDMM e MC) dispuseram informações dos prontuários de todos os pacientes com diagnóstico de ceratocone por eles transplantados que preenchiam os critérios de inclusão.

Os pacientes transplantados por 27 médicos em treinamento foram selecionados a partir do arquivo do Setor de Doenças Externas e Córnea do Departamento de Oftalmologia da UNIFESP-EPM, tendo sido inscritos para transplante de córnea no período de maio de 1993 a dezembro de 1998.
Cada prontuário foi examinado e seus dados recolhidos. Os prontuários que não possuíam dados completos de interesse para o estudo foram rejeitados. Explicou-se aos pacientes o objetivo do estudo e, em seguida, agendou-se uma consulta para aqueles que concordaram em participar.

Setenta olhos de setenta pacientes com transplante de córnea por ceratocone realizados por médicos experientes (Grupo I) e em treinamento (Grupo II) foram examinados. Considerou-se "médico experiente" aquele com experiência anterior em, pelo menos, 100 (cem) cirurgias de transplante de córnea e "médico em treinamento" o cirurgião, residente do terceiro ano ou estagiário, em treinamento no Setor de Doenças Externas e Córnea do Departamento de Oftalmologia da UNIFESP-EPM.

As informações prévias ao exame clínico realizado neste trabalho, colhidas dos prontuários dos pacientes, incluíam os dados sobre o exame clínico de abertura do prontuário, ato cirúrgico e complicações pós-operatórias. O diagnóstico de ceratocone foi derivado da observação de uma ou mais variáveis a seguir: ectasia corneal, sinal de Musson, linhas de Vogt, anel de Fleischer, opacidades superficiais ou mais profundas em casos com história pregressa de hidropisia (sinais estes encontrados no exame à lâmpada de fenda), bem como das informações sobre topografia e ou ceratometria.

As cirurgias de ambos os grupos foram realizadas, seguindo, dentro do possível, um mesmo padrão.

As córneas doadoras foram trepanadas com trépanos manuais por via endotelial. No receptor, a trepanação foi realizada centralmente na córnea, incluindo a base do cone. A câmara anterior foi reformada com material visco-elástico, hialuronato de sódio ou hidroxipropilmetil celulose $2 \%$. Com exceção de dois transplantes, todas as córneas foram suturadas com pontos separados com fio de mononylon 10-0. No grupo I, uma cirurgia foi realizada com sutura contínua e outra com sutura mista. A terapêutica pós-operatória utilizada incluiu uma combinação de antibiótico e esteróide na forma de colírio, com frequiência, duração e concentração individualizada para cada paciente. Iniciou-se a remoção seletiva das suturas para redução do astigmatismo três meses após a cirurgia. Quando o astigmatismo atingia proporções aceitáveis $(\leq 3 \mathrm{D})$, as demais suturas eram deixadas in situ até que se rompessem ou afrouxassem espontaneamente.

Com referência às complicações pós-operatórias, considerou-se falência primária, a ausência irreversível de transparência do enxerto ocorrendo nas duas primeiras semanas após a cirurgia, em razão das características do tecido doador e não relacionada ao perfil do paciente que recebeu o enxerto. Em casos de falência primária, os olhos eram transplantados novamente, mas as cirurgias apenas foram consideradas retransplantes se tivessem que ser substituídas por outras causas de falência.

A falência secundária do enxerto poderia ter diversas causas, sendo considerada qualquer alteração do enxerto, que era inicialmente claro por pelo menos duas semanas após a cerato- 
plastia, tendo a alteração impedido a recuperação da visão útil, e tendo sido refratária a todas as opções de tratamento.

Os pacientes foram submetidos ao exame clínico oftalmológico por um único observador (VLDMM) com os mesmos aparelhos, no mesmo lugar e condições técnicas. A análise da forma e padrões topográficos foi realizada por três oftalmologistas independentes.

O exame clínico oftalmológico consistiu na obtenção da anamnese, ectoscopia, avaliação da acuidade visual (AV) com e sem correção, exame biomicroscópico do segmento anterior ocular, medida da pressão intra-ocular (PIO), fundoscopia, refratometria dinâmica e análise topográfica. A videoceratoscopia computadorizada foi realizada com o aparelho EyeSys (EyeSys Corneal Analysis System 2000 software version 3.1, EyeSys Technologies, Inc - Houston TP, EUA).

O resultado da AV corrigida, medida por meio da tabela de Snellen, foi convertido em medidas logarítmicas do ângulo mínimo de resolução (LogMAR). A AV descrita como "contadedos" foi substituída pelo equivalente numérico de 0,02 $(\operatorname{LogMAR} 1,70)^{(19)}$.

Para análise dos erros refrativos e dos resultados da videoceratoscopia computadorizada utilizou-se um tratamento matemático vetorial, cujo objetivo foi representar os dados em um espaço dióptrico tridimensional. Dessa forma, os três componentes cartesianos ortogonais $(\mathrm{x}, \mathrm{y}$ e $\mathrm{z})$ foram objeto de avaliações e análises estatísticas. Adotou-se a representação vetorial, oriunda da análise de Fourier do perfil do poder dióptrico das lentes esferocilíndricas ${ }^{(20)}$.

O valor do módulo do componente cilíndrico da refração (DC) sem tratamento vetorial também foi avaliado.

Para a análise da videoceratoscopia computadorizada foi usado o mapa axial de cores, em que foram avaliados os padrões e as formas topográficas. Os critérios empregados para a análise foram os sugeridos por Karabatsas et al. ${ }^{(21)}$ e Sharma et al. ${ }^{(22)}$.

Com a finalidade de avaliar a funcionalidade dos enxertos de córnea neste estudo, escolheu-se quatro desfechos: componente esférico da refração (RE), componente cilíndrico da refração (DC), topografia e acuidade visual corrigida com ócu- los. Esses resultados foram avaliados e comparados segundo a experiência do cirurgião.

\section{Testes estatísticos}

A presença de associação entre parâmetros qualitativos foi avaliada pelo teste do Qui-quadrado ou teste exato de Fisher.

Para testar a hipótese de normalidade da amostra empregou-se o teste de Kolmogorov-Smirnov. Como a maior parte dos parâmetros não tinha distribuição normal evidenciada, utilizou-se testes não-paramétricos em todas as análises.

A comparação entre grupos independentes em relação às variáveis quantitativas foi feita pela prova de Mann-Whitney, quando se tratavam de duas amostras, e pela prova de Kruskal-Wallis para três ou mais amostras.

Avaliou-se a presença de correlação entre variáveis quantitativas pelo coeficiente de correlação proposto de Spearman $\left(r_{s}\right)$.

O nível de significância de $0,05(\alpha=5 \%)$ foi adotado, e os níveis descritivos (p) iguais ou inferiores a esse valor foram considerados significantes e representados por *.

\section{RESULTADOS}

A amostra constituiu-se de 70 olhos; sendo 32 (45,7\%) olhos direitos e $38(54,3 \%)$ olhos esquerdos. A média de idade dos pacientes foi de 35,8 anos $(\mathrm{dp}=11,7)$ e mediana de 35 anos, variando entre 20 e 75 anos. Trinta e cinco (50\%) eram femininos e $35(50 \%)$ masculinos. A média de idade dos pacientes na ocasião do transplante foi de 28,2 anos $(\mathrm{dp}=11)$ e mediana de 26,3 anos, variando entre 11 e 65 anos. A média de tempo transcorrido entre a cirurgia e a avaliação foi de 7,9 anos $(d p=3,5)$, variando entre 1,6 e 20 anos. Em todos estes parâmetros não foi encontrada diferença estatisticamente significante entre os dois grupos $(\mathrm{p}>0,05)$.

Os dados referentes à técnica cirúrgica encontram-se na tabela 1 .

Os dados referentes às complicações pós-operatórias encontram-se na tabela 2 .

\begin{tabular}{|c|c|c|c|}
\hline Parâmetros cirúrgicos & Experiente & Treinamento & Comparação \\
\hline \multicolumn{4}{|c|}{ Diâmetro de trepanação $(\mathrm{mm})$} \\
\hline Média $\pm \mathrm{dp}$ & $8,01 \pm 0,20$ & $7,90 \pm 0,27$ & \multirow{3}{*}{$p=0,108$} \\
\hline Variação & $7,50-8,50$ & $7,25-8,50$ & \\
\hline Mediana (n) & $8,00(34)$ & $8,00(26)$ & \\
\hline \multicolumn{4}{|c|}{ Diferença entre doador e receptor $(\mathrm{mm})$} \\
\hline Média \pm dp & $0,26 \pm 0,15$ & $0,25 \pm 0,12$ & \multirow{3}{*}{$p=0,670$} \\
\hline Variação & $0,00-0,50$ & $0,00-0,50$ & \\
\hline Mediana (n) & $0,25(34)$ & $0,25(26)$ & \\
\hline \multicolumn{4}{|l|}{ Tipo de sutura - n (\%) } \\
\hline $\mathrm{C}$ & $1(2,9)$ & $0(0,0)$ & \multirow{3}{*}{$p=0,357$} \\
\hline I & $33(94,3)$ & $35(100,0)$ & \\
\hline M & $1(2,9)$ & $0(0,0)$ & \\
\hline
\end{tabular}


No grupo de cirurgiões em treinamento foi encontrada maior proporção de ocorrência de rejeição endotelial, com diferença estatisticamente significante entre os grupos $(\mathrm{p}=0,006)$.

A acuidade visual pré-operatória dos pacientes mostrouse significantemente melhor no grupo de cirurgiões experientes $(\mathrm{p}<0,001)$ (Tabela 3$)$.

$\mathrm{Na}$ análise dos prontuários foram encontrados 7 (10\%) olhos com hipertensão ocular e, à época do exame, quando já não faziam uso de corticosteróide, $4(5,7 \%)$ pacientes ainda faziam uso de medicação anti-hipertensiva e apenas um deles tinha $\mathrm{PIO}$ acima de $21 \mathrm{mmHg}$. Em nenhum dos pacientes, foi observado aumento da escavação do disco óptico.

Com relação à avaliação clínica pós-operatória, os dados encontram-se na tabela 3 .

Ao exame clínico, alta miopia ocorreu em sete olhos (10\%). Porém, degeneração fundoscópica responsável por baixa acui-

\begin{tabular}{|c|c|c|c|}
\hline Complicações & Experiente & Treinamento & Comparação \\
\hline Falência primária & $1(2,9 \%)$ & $1(2,9 \%)$ & $p=1,000$ \\
\hline Ressutura & $0(0,0 \%)$ & $1(2,9 \%)$ & $p=0,314$ \\
\hline Infecção (Ponto) & $1(2,9 \%)$ & $0(0,0 \%)$ & $p=0,314$ \\
\hline Rejeição endotelial & $2(5,7 \%)$ & $11(31,4 \%)$ & $p=0,006^{\star}$ \\
\hline Hipertensão intra-ocular & $3(8,6 \%)$ & $4(11,4 \%)$ & $p=0,690$ \\
\hline Catarata & $1(2,9 \%)$ & $1(2,9 \%)$ & $p=1,000$ \\
\hline
\end{tabular}

\begin{tabular}{|c|c|c|c|}
\hline Parâmetros & Experiente & Treinamento & Comparação \\
\hline \multicolumn{4}{|c|}{ AV pré-operatória em LogMAR } \\
\hline Média \pm dp & $1,25 \pm 0,37$ & $1,63 \pm 0,25$ & \multirow{3}{*}{$\mathrm{p}<0,001^{*}$} \\
\hline Mínimo - máximo & $0,52-1,70$ & $0,52-1,70$ & \\
\hline Mediana (n) & $1,30(35)$ & $1,70(35)$ & \\
\hline \multicolumn{4}{|l|}{ AV s/c em LogMAR } \\
\hline Média \pm dp & $0,858 \pm 0,540$ & $0,904 \pm 0,596$ & \multirow{3}{*}{$p=0,894$} \\
\hline Mínimo - máximo & $0,18-1,70$ & $0,00-1,70$ & \\
\hline Mediana (n) & $0,70(35)$ & $0,85(35)$ & \\
\hline \multicolumn{4}{|l|}{ AV c/c em LogMAR } \\
\hline Média \pm dp & $0,235 \pm 0,308$ & $0,350 \pm 0,519$ & \multirow{3}{*}{$p=0,943$} \\
\hline Mínimo - máximo & $0,00-1,70$ & $-0,12-1,70$ & \\
\hline Mediana (n) & $0,18(35)$ & $0,18(35)$ & \\
\hline \multicolumn{4}{|l|}{ MOE - n (\%) } \\
\hline ET & $2(5,7)$ & $1(2,8)$ & \multirow{3}{*}{$p=0,641$} \\
\hline $\mathrm{N}$ & $30(85,7)$ & $29(82,9)$ & \\
\hline $\mathrm{XT}$ & $3(8,6)$ & $5(14,3)$ & \\
\hline \multicolumn{4}{|l|}{ sFO - n (\%) } \\
\hline Normal & $31(88,6)$ & $29(90,6)$ & \multirow[t]{2}{*}{$p=0,720$} \\
\hline Alta miopia & $4(11,4)$ & $3(8,8)$ & \\
\hline \multicolumn{4}{|l|}{ Vascularização n (\%) } \\
\hline Sem vascularização & $29(91,5)$ & & \multirow[t]{2}{*}{$p=0,001$} \\
\hline Vascularizado & $6(8,5)$ & $19(27,1)$ & \\
\hline \multicolumn{4}{|c|}{ Transparência da córnea - n (\%) } \\
\hline $\mathrm{C}$ & $34(97,1)$ & $28(80,0)$ & \multirow{3}{*}{$p=0,061$} \\
\hline $\mathrm{H}$ & $0(0,0)$ & $4(11,4)$ & \\
\hline $\mathrm{O}$ & $1(2,9)$ & $3(8,6)$ & \\
\hline \multicolumn{4}{|l|}{ Tonometria } \\
\hline Média $\pm \mathrm{dp}$ & $13,8 \pm 1,9$ & $13,7 \pm 2,9$ & \multirow{3}{*}{$p=0,596$} \\
\hline Mínimo - máximo & $10-19$ & $8-24$ & \\
\hline Mediana (n) & $14(35)$ & $13(35)$ & \\
\hline
\end{tabular}




\begin{tabular}{|c|c|c|c|}
\hline Parâmetros - refração & Experiente & Treinamento & Comparação \\
\hline \multicolumn{4}{|l|}{ M - equivalente esférico } \\
\hline Média \pm dp & $-3,22 \pm 3,70$ & $-4,38 \pm 6,22$ & \multirow{3}{*}{$p=0,826$} \\
\hline Mínimo - máximo & $-14,00-3,38$ & $-21,75-3,88$ & \\
\hline Mediana (n) & $-2,75 \quad(35)$ & $-2,06 \quad(32)$ & \\
\hline \multicolumn{4}{|l|}{ Refração J0 } \\
\hline Média $\pm \mathrm{dp}$ & $-0,13 \pm 1,35$ & $0,01 \pm 1,07$ & \multirow{3}{*}{$p=0,555$} \\
\hline Mínimo - máximo & $-2,85-2,49$ & $-1,86-1,80$ & \\
\hline Mediana (n) & $-0,06 \quad(35)$ & $-0,06 \quad(32)$ & \\
\hline \multicolumn{4}{|l|}{ Refracão J45 } \\
\hline Média $\pm \mathrm{dp}$ & $-0,14 \pm 1,18$ & $-0,21 \pm 1,25$ & \multirow{3}{*}{$p=0,735$} \\
\hline Mínimo - máximo & $-2,53-2,31$ & $-2,69-2,21$ & \\
\hline Mediana (n) & 0 & $-0,27 \quad(32)$ & \\
\hline $\mathrm{dp}=$ desvio-padrão; $\mathrm{n}=$ número & & & \\
\hline
\end{tabular}

dade visual relacionada à alta miopia ocorreu em cinco olhos, semelhante nos dois grupos. Vascularização corneal foi observada em maior número de olhos no Grupo II, com diferença estatística $(\mathrm{p}=0,001)$ quando comparada ao Grupo I. Quanto à alteração da motricidade extrínseca ocular, apenas um olho com ambliopia entrou na amostra. Falência do enxerto ocorreu em três olhos, dois após rejeição e um após falência primária, não tendo sido reoperado. Todas as falências ocorreram no Grupo II.

Os resultados da média, desvio-padrão e mediana dos componentes vetoriais (M, J0 e J45) da refração (Tabela 4) e os resultados reconvertidos para a notação esferocilíndrica, considerando-se todos os olhos da amostra, divididos segundo a experiência do cirurgião, encontram-se nos dados da tabela 5 . Não foi encontrada diferença estatisticamente significante entre os grupos I e II.

Os resultados da videoceratoscopia computadorizada na notação vetorial (Tabela 6) e os resultados reconvertidos para a notação esferocilíndrica, divididos nos dois grupos, encontram-se na tabela 7. Não foi observada diferença estatística entre ambos.

As análises qualitativas dos mapas topográficos foram realizadas por três oftalmologistas independentes. Todos os padrões assinalados tiveram a concordância de, no mínimo, dois examinadores. Não foi encontrada associação estatisticamente significante entre a experiência do cirurgião e os resultados da videoceratoscopia computadorizada nos quesitos forma $(\mathrm{p}=0,244)$ ou padrão $(\mathrm{p}=0,302)$.

O padrão topográfico encontrado mais comumente foi o regular $(73,1 \%)$ e em 19,4\% observou-se o padrão irregular, sem diferença estatisticamente significante entre os grupos I e II. Entre os padrões regulares, houve praticamente o mesmo número de topografias com padrões simétrico e assimétrico, $40,3 \%$ e $32,8 \%$, respectivamente.

Com relação à acuidade visual pós-operatória corrigida dos pacientes, considerando-se todos os olhos da amostra, não houve diferença estatisticamente significante confrontada com a experiência do cirurgião $(p>0,05)$ em todas as compa- rações (Tabela 3 ). A tabela 8 mostra o resultado da AV corrigida e a comparação entre os dois grupos, tendo sido removidos da amostra os olhos que apresentam outras alterações (degeneração retínica, ambliopia, etc.) que interferem com o resultado do transplante de córnea.

A AV pré-operatória não mostrou correlação estatisticamente significante quando comparada com a pós-operatória (rs= $-0,167 ; p=0,179)$.

\section{DISCUSSÃO}

Embora, na literatura, tenham sido encontrados bons resultados após ceratoplastia, o transplante penetrante de córnea ainda não é indicado como primeira opção no tratamento do ceratocone, sendo esta modalidade de tratamento reservada para uma minoria de pacientes ${ }^{(23-25)}$.

Optou-se por fazer um estudo comparativo dos resultados dos transplantes realizados por cirurgiões experientes (Grupo I)

\begin{tabular}{|c|c|c|}
\hline Parâmetros - refração & Experiente & Treinamento \\
\hline \multicolumn{3}{|l|}{ Esférico } \\
\hline Média \pm dp & $-3,03 \pm 5,49$ & $-4,17 \pm 7,87$ \\
\hline Mínimo - máximo & $-12,00-+4,00$ & $-20,00-+6,50$ \\
\hline Mediana (n) & $-2,69(35)$ & $-1,78(32)$ \\
\hline \multicolumn{3}{|l|}{ Cilíndrico } \\
\hline Média $\pm \mathrm{dp}$ & $-0,38 \pm 3,59$ & $-0,42 \pm 3,29$ \\
\hline Mínimo - máximo & $-7,50--0,75$ & $-5,75-0,00$ \\
\hline Mediana (n) & $-0,12(35)$ & $-0,55(32)$ \\
\hline \multicolumn{3}{|l|}{ Eixo } \\
\hline Média $\pm \mathrm{dp}$ & $114 \pm 21$ & $136 \pm 25$ \\
\hline Mínimo - máximo & $5-180$ & $10-180$ \\
\hline Mediana (n) & $90(35)$ & $129(32)$ \\
\hline
\end{tabular}




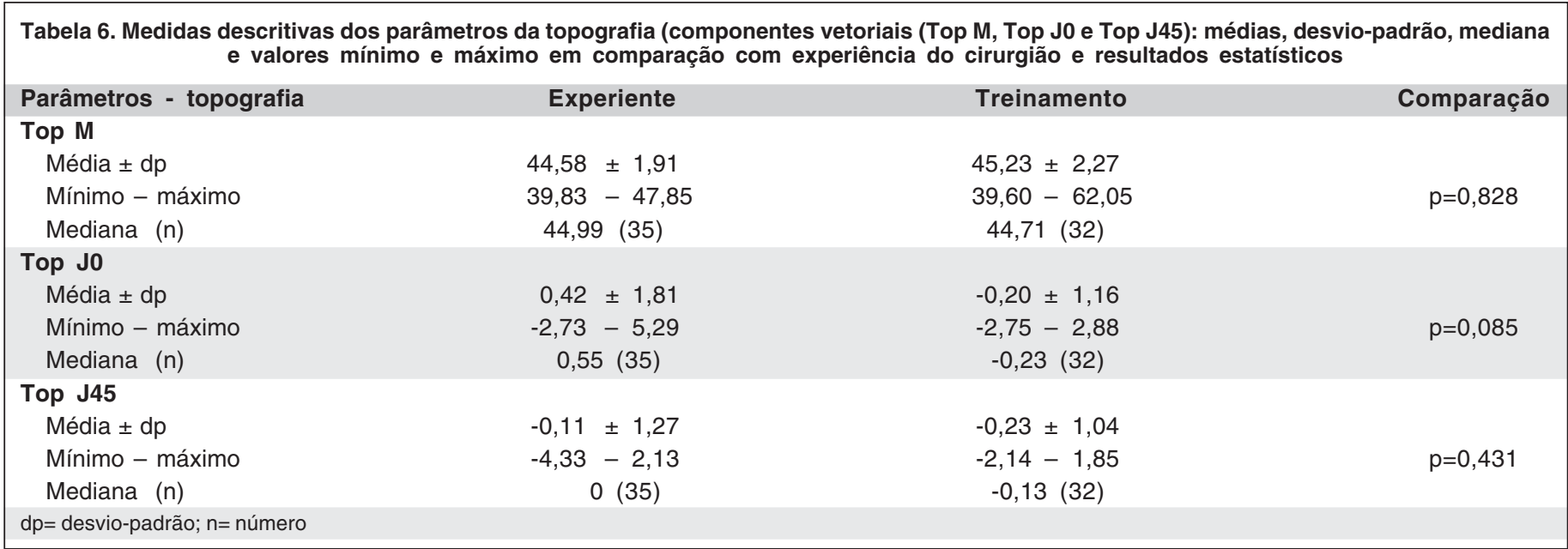

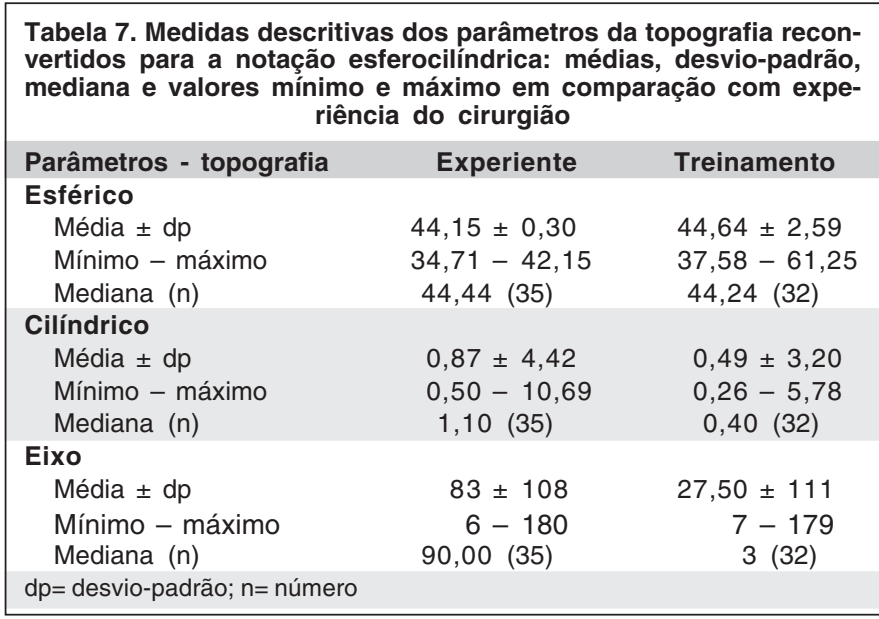

\begin{tabular}{|c|c|c|c|}
\hline \multicolumn{4}{|c|}{$\begin{array}{l}\text { Tabela 8. Relaciona a variável experiência do cirurgião com o } \\
\text { parâmetro acuidade visual com correção, considerando todos os } \\
\text { olhos exceto os que tenham baixa visão por outros motivos que o } \\
\text { transplante de córnea e os resultados estatísticos }\end{array}$} \\
\hline Parâmetros & $\begin{array}{l}\text { Cirurgião } \\
\text { experiente } \\
(n=32)\end{array}$ & $\begin{array}{l}\text { Cirurgião em } \\
\text { treinamento } \\
\quad(n=28)\end{array}$ & Comparação \\
\hline \multicolumn{4}{|l|}{ AV com correção } \\
\hline Média $\pm \mathrm{dp}$ & $0,17 \pm 0,15$ & $0,12 \pm 0,13$ & \\
\hline Mediana & 0,14 & 0,10 & $p=0,227$ \\
\hline Mínimo / máximo & $0,00 / 0,48$ & $-0,12 / 0,40$ & \\
\hline
\end{tabular}

e cirurgiões em treinamento (Grupo II), por conta da variabili, sinais de ceratocone avançado ${ }^{(25)}$. Isso pode explicar porque as cirurgias, quando indicadas, ocorrem na faixa etária relativamente jovem, e, na presente amostra, não foi diferente ${ }^{(2,16,26-29)}$.

Neste estudo, não houve diferença quanto ao número de pacientes do sexo masculino e feminino participantes, embora na literatura haja predominância do sexo masculino entre os indivíduos portadores de ceratocone que se submetem à cirurgia $^{(2,30)}$. Considerando que a incidência do ceratocone seja igual em ambos os sexos ${ }^{(25)}$, imagina-se que o ceratocone seja uma doença mais rapidamente progressiva no sexo masculino ou que os homens tenham maior tendência a procurar uma solução cirúrgica do que as mulheres ${ }^{(31)}$. Entretanto, essa hipótese não é compartilhada por outros autores ${ }^{(32)}$.

Embora enxertos com diâmetros pequenos $(<7,0 \mathrm{~mm}) \mathrm{e}$ grandes $(>8,5 \mathrm{~mm})$ possam ser usados ocasionalmente, a maioria dos cirurgiões usa trépanos que variam entre 7,0 a 8,5 mm de diâmetro ${ }^{(33)}$. Sem diferença entre os grupos, neste estudo, os diâmetros de trepanação da córnea receptora empregados estiveram entre 7,50 a 8,50 mm.

As técnicas de sutura figuram entre os mais importantes fatores relacionados ao astigmatismo pós-operatório e vários estilos diferentes de suturas têm sido usados e estudados ${ }^{(34-35)}$. Alguns autores escolhem o método de sutura influenciados pelas características do olho receptor, e suturas isoladas freqüentemente são selecionadas para receptores de alto risco ${ }^{(36-37)}$.

No presente estudo, o tipo de sutura mais utilizado, quer seja entre os cirurgiões experientes, quer seja pelos cirurgiões em treinamento, foi a isolada $(97,1 \%)$ sem diferença entre os grupos de cirurgiões. No Serviço onde foram realizadas as cirurgias do grupo II, as suturas isoladas são as recomendadas aos cirurgiões em treinamento e as de escolha em casos em que as córneas apresentam afinamento corneal; embora se trate de opção pessoal, quando o cirurgião já possui experiência.

Embora o transplante de córnea seja o mais antigo, mais comum e a forma de transplante de tecido de mais sucesso, em razão do privilégio imunológico da câmara anterior ${ }^{(38)}$, a rejeição continua a ser a maior causa de falência nos transplantes de córnea ${ }^{(39)}$, mesmo considerando córneas não vascularizadas como é o caso do ceratocone ${ }^{(40)}$.

Vários fatores de risco para a rejeição, após transplante de córnea, têm sido estabelecidos por estudos multicêntricos e prospectivos, tais como: o Corneal Transplant Follow-up Stu$d y^{(41)}$, o Collaborative Corneal Transplantation Study Re- 
search Group ${ }^{(17,42)}$ e o Australian Corneal Graft Registry ${ }^{(43)}$. Os mais citados são: idade menor do que 40 anos $^{(42)}$, sinéquias anteriores periféricas ${ }^{(37)}$, neovascularização corneal ${ }^{(36,44-45)}$, inflamação corneal preexistente ${ }^{(43)}$, sensibilização a antígenos de histocompatibilidade estranho pelo enxerto do olho contralateral em casos de bilateralidade do enxerto ${ }^{(46)}$, diâmetro de enxerto acima de $8,5 \mathrm{~mm}^{(30)}$ ou menor do que $7,5 \mathrm{~mm}^{(46)}$, enxerto anterior $^{(42,47)}$ e suturas frouxas ${ }^{(45)}$.

Neste estudo, foram observadas 28 reações de rejeição de todos os tipos (epitelial, subepitelial, endotelial e não caracterizada) em 19 olhos $(27,1 \%)$. Número relativamente grande, considerando ser o grupo de pacientes com pouca história de inflamação ocular. Destes, apenas dois casos $(2,8 \%)$ evoluíram para falência do enxerto.

Wilhelmus, discutindo o artigo de Epstein et al., confeccionou uma tabela com 25 séries selecionadas de ceratoplastias penetrantes por ceratocone e suas respectivas porcentagens de rejeição. O índice de reação de rejeição naquelas séries variou de 6\% a 57\% com uma média de 19,68\% ${ }^{(48)}$. A série de Epstein et al. foi a maior, com 345 transplantes por ceratocone, $18,5 \%$ de rejeição nos primeiros transplantes por ceratocone e apenas nove falências por rejeição $(2,42 \%)^{(48)}$. Observa-se praticamente a mesma taxa de falência por rejeição no presente estudo.

Talvez o trauma cirúrgico possa gerar inflamação suficiente para provocar sensibilização, bem como a diferença na porcentagem de rejeição entre os vários estudos pode ser atribuída às variações na técnica cirúrgica ao longo do tempo e entre cirurgiões experientes e em treinamento.

No presente estudo, seis olhos $(8,6 \%)$ tiveram rejeição e, por hipótese, poderiam ter sido sensibilizados previamente pelo enxerto no olho contralateral, enquanto $13(18,6 \%)$ olhos não foram sensibilizados por um enxerto anterior. Nesta amostra, não se pôde estabelecer as causas dos referidos episódios, uma vez que dados, como o da vascularização, importante fator de risco, só foram determinados posteriormente ao episódio de rejeição por ocasião do exame clínico. Embora a rejeição seja uma complicação que afeta elevada porcentagem de transplantes de córnea, a menos que o episódio de rejeição conduza à falência do enxerto, não se observa efeito adverso na acuidade visual ${ }^{(2,45)}$.

No presente estudo foi encontrada diferença estatisticamente significante entre os grupos no parâmetro rejeição endotelial $(\mathrm{p}=0,006)$, sendo que o grupo II apresentou maior taxa de rejeição endotelial. Chalita et al. estudaram a rejeição corneal em 113 transplantes de córnea ópticos eletivos por diferentes indicações, realizados no Departamento de Oftalmologia da UNIFESP-EPM, em 1998. Observaram 17,69\% de rejeição do botão transplantado e creditaram esta taxa ao fato de as cirurgias terem sido realizadas por cirurgiões em treinamento, com menor experiência, e, portanto, com maior tempo cirúrgico e maior trauma do tecido, além do fato de esse Serviço ser considerado terciário, para onde são encaminhados os casos complexos e de prognóstico mais reservado ${ }^{(49)}$.

Glaucoma é tido como importante fator de risco para falência do transplante de córnea ${ }^{(36-37,42)}$. Em seu estudo sobre fatores de risco para falência do enxerto, Boisjoly et al. encontraram uma forte associação de glaucoma com falência sem reação imune prévia ${ }^{(36)}$. Estes autores formularam a hipótese de que a falência poderia estar relacionada à flutuação da PIO com picos que, repetidamente, danificariam o endotélio da córnea doadora. Pacientes usuários de medicação anti-glaucomatosa têm também um risco aumentado de rejeição e de falências relacionadas a alterações da superfície ocular em razão do acúmulo de células de resposta inflamatória na conjuntiva, secundária ao uso tópico desse tipo de medicação com conservantes ${ }^{(37)}$. Na maioria dos olhos com transplante de córnea sem história prévia de glaucoma, tal como no ceratocone, a pressão intra-ocular raramente é um problema. Se há aumento da pressão intra-ocular, esta pode ser devida ao uso prolongado de corticosteróide, uveíte persistente ou dano no mecanismo de drenagem do aquoso ${ }^{(50)}$.

Dessas observações, depreende-se que os motivos que ocasionam o aumento da pressão intra-ocular em olhos submetidos a transplante de córnea, fácicos, sem procedimentos que causem danos às estruturas intra-oculares, particularmente à câmara anterior e que sejam de diâmetro médio de $8 \mathrm{~mm}$, devem ser muito poucos.

Com relação à hipertensão intra-ocular relacionada à experiência do cirurgião, não foi encontrada diferença estatística entre os grupos (Tabela 3). Esse resultado difere de estudo realizado por Cavalcanti et al. em que o glaucoma pós-operatório foi a complicação mais freqüente, acometendo $26,1 \%$ dos olhos com ceratocone, operados por residentes ${ }^{(51)}$.

Kutzscher et al., em seu estudo sobre os resultados de ceratoplastia penetrante realizada por residentes, também encontrou a PIO elevada como complicação mais comum (25\%); mas, nessa série, analisaram transplantes indicados por todas as causas e a maioria (7/10) possuía pelo menos um fator de risco para essa complicação, ou seja, glaucoma pré-operatório, afacia, inflamação do segmento anterior, vitrectomia, remoção da lente intra-ocular e trauma pré-operatório ${ }^{(52)}$.

O uso estendido do corticosteróide tópico, trauma de uma cirurgia intra-ocular e reação inflamatória são fatores que aumentam o risco do aparecimento de opacidade do cristalino em indivíduos jovens. Cavalcanti et al. verificaram $10,9 \%$ de incidência de catarata em uma amostra de 43 pacientes com ceratocone $^{(51)}$, enquanto no presente estudo catarata foi referida por $2(2,8 \%)$ pacientes, após o transplante de córnea, um de cada grupo (Tabela 2).

Pode-se dizer que o presente estudo comportou-se como outros da literatura ${ }^{(53)}$, em que, a reação imune foi a única complicação de significância.

A maioria dos estudos reporta os resultados refrativos, seguindo transplante de córnea, em um período de 18 meses a seis anos de pós-operatório ${ }^{(28,54)}$. Resultados de pós-operatórios tardios tendem a estabelecer mais a razão de sobrevivência do que de resultados refrativos ${ }^{(55)}$.

Optou-se pela análise dos olhos transplantados após todas as suturas terem sido removidas, pois é sabido que com a remoção das mesmas, na maior parte dos casos, existe uma 
seis anos de pós-operatório ${ }^{(28,54)}$. Resultados de pós-operatórios tardios tendem a estabelecer mais a razão de sobrevivência do que de resultados refrativos ${ }^{(55)}$.

Optou-se pela análise dos olhos transplantados após todas as suturas terem sido removidas, pois é sabido que com a remoção das mesmas, na maior parte dos casos, existe uma alteração das curvaturas corneais com grande variabilidade na refração esférica, bem como cilíndrica ${ }^{(56-57)}$.

De acordo com os critérios de inclusão deste estudo, os olhos submetidos a transplante de córnea por ceratocone que apresentassem outras alterações oculares, exceto a ceratoplastia, não deveriam ser admitidos na amostra. Todavia, a miopia parece ser uma condição de certa forma relacionada aos olhos com ceratocone. Entretanto, miopias elevadas podem vir associadas a alterações fundoscópicas que impedem, por exemplo, a análise dos resultados da acuidade visual devida somente ao transplante de córnea.

Observa-se que houve diferença nos resultados da ametropia esférica quando os dois grupos de experiência do cirurgião foram comparados, sendo que o Grupo I apresentou miopia menor do que o Grupo II, porém, esta diferença não teve significância estatística (Tabelas 4 e 5). A comparação desses resultados com outras séries publicadas, somente incluindo resultados de transplante de córnea por ceratocone, encontra-se no quadro 1.

O astigmatismo pós-operatório elevado é um dos obstáculos para a reabilitação visual. No presente estudo observou-se que houve diferença nos resultados do astigmatismo, conforme a experiência do cirurgião. $\mathrm{O}$ astigmatismo resultante dos transplantes de córnea operados por cirurgiões experientes foi discretamente menor do que aquele resultante do grupo de cirurgiões em treinamento, porém, sem diferença estatística entre ambos (Tabelas 4 e 5).

Estudando outras séries de transplantes por ceratocone, a média do astigmatismo reportada variou entre 1,20 e 5,00 D, após todas as suturas terem sido removidas, usando uma variedade de técnicas cirúrgicas ${ }^{(2,28,52,58-64)}$. Os resultados desses estudos em comparação com o presente, incluindo os dos Grupos I e II, encontram-se no quadro 2.

Com o objetivo de poder relacionar os resultados obtidos neste estudo com os descritos na literatura, também foi analisado o valor do astigmatismo sem consideração de seu respectivo eixo (módulo). Foi obtida a média de $-3,36 \mathrm{DC}( \pm 1,72$ DC) e a mediana de -3,25 DC (Quadro 2). Observa-se que os resultados obtidos de ambos os grupos são comparáveis aos encontrados na literatura.

A análise topográfica computadorizada tem contribuído para aumentar o entendimento de muitas doenças ${ }^{(65)}$. Essa análise tem sido útil no esclarecimento das alterações que ocorrem na córnea após manipulação cirúrgica, incluindo ceratoplastia penetrante ${ }^{(56,66)}$

No presente estudo, não houve diferença entre os dois grupos considerando-se a análise dos resultados quantitativos da videoceratoscopia computadorizada (Tabelas 6 e 7).

\begin{tabular}{|c|c|c|}
\hline Autor & Refração esférica (DE) & Intervalo \\
\hline Kirkness et al. ${ }^{(16)}$ & $-2,68$ & $-16,00 a+4,00$ \\
\hline Brierly et al. ${ }^{(2)}$ & $-4,09 \pm 3,80$ & \\
\hline Tuft et al. ${ }^{(74)}$ & $-4,83$ & $-19,50 a+3,40$ \\
\hline Watson et al. ${ }^{(7)}$ & $-1,63$ & $-16,00 a+4,00$ \\
\hline Spadea et al. ${ }^{(61)}$ & $-0,49 \pm 3,70 \mathrm{EE}$ & \\
\hline Brahma et al. ${ }^{(29)}$ & $-3,75$ & $-10,00 a+4,00$ \\
\hline Buzard et al. ${ }^{(62)}$ & $-1,70 \pm 3,00 \mathrm{EE}$ & \\
\hline Doyle et al. ${ }^{(3)}$ & $-2,50$ & $-13,50 a+1,00$ \\
\hline Mascaro et al. & $-3,59 \pm 6,77$ & $-20,00 a+6,50$ \\
\hline Grupo I & $-3,03 \pm 5,49$ & $-12,00 a+4,00$ \\
\hline Grupo II & $-4,17 \pm 7,87$ & $-20,00 a+6,50$ \\
\hline
\end{tabular}

\begin{tabular}{|c|c|c|}
\hline \multicolumn{3}{|c|}{$\begin{array}{l}\text { Quadro 2. Resultado da média da refração astigmática dos trans- } \\
\text { plantes de córnea por ceratocone }\end{array}$} \\
\hline Autor & Refração cilíndrica & (DC) - Vetorial \\
\hline Troutman, Gaster ${ }^{(59)}$ & 3,80 a 4,10 & \\
\hline Brierly et al. ${ }^{(2)}$ & $-2,67 \pm 2,04$ & \\
\hline Lim et al. ${ }^{(28)}$ & $-5,00 \pm 3,00$ & \\
\hline Jensen e Maumenee ${ }^{(58)}$ & 3,35 a 4,62 & \\
\hline Spadea et al. ${ }^{(61)}$ & $-1,20 \pm 3,11$ & \\
\hline Kutzscher et al. ${ }^{(52)}$ & $3,40 \pm 2,10$ & \\
\hline Buzard et al. ${ }^{(62)}$ & $-3,10 \pm 1,70$ & \\
\hline Olson et al. ${ }^{(63)}$ & $2,76 \pm 1,99$ & \\
\hline Dolorico et al. ${ }^{(64)}$ & $3,11(0,00$ a 9,00$)$ & \\
\hline De Lavalette et al. ${ }^{(60)}$ & $4,70(0,00$ a 9,00$)$ & \\
\hline Mascaro et al & $-0,39 \mathrm{DC} \pm 3,44 \mathrm{DC}$ & $(0,00$ a $-7,50)$ \\
\hline Módulo & $-3,36 \mathrm{DC} \pm 1,72 \mathrm{DC}$ & $(0,00$ a $-7,50)$ \\
\hline Grupo I & $-0,38 D C \pm 3,59 D C$ & $(-7,50$ a $-0,75)$ \\
\hline Módulo & $-3,62 \mathrm{DC} \pm 1,66 \mathrm{DC}$ & $(-7,50$ a $-0,75)$ \\
\hline Grupo II & $-0,42 \mathrm{DC} \pm 3,29 \mathrm{DC}$ & $(-5,75$ a 0,00$)$ \\
\hline Módulo & $-3,16 \mathrm{DC} \pm 1,79 \mathrm{DC}$ & $(-5,75$ a 0,00$)$ \\
\hline
\end{tabular}

Com relação ao padrão topográfico, observa-se que o mesmo resultado foi encontrado por outros autores que verificaram aumento na proporção de miras ceratométricas regulares após a remoção das suturas ${ }^{(57,67)}$.

O resultado óptico, após transplante de córnea, mensurado por índices convencionais, tais como: transparência do enxerto e ceratometria, nem sempre é paralelo com o resultado visual.

Neste estudo, foi observada diferença estatística relacionada com a acuidade visual pré-operatória em que se observa que os pacientes com indicação de transplante de córnea para correção do ceratocone no Grupo I, de médicos experientes, apresentam acuidade visual melhor do que os do Grupo II (Tabela 3).

Parece razoável que um serviço terciário, como é o caso do Setor de Doenças Externas e Córnea do Departamento de Oftalmologia da UNIFESP- EPM, seja procurado por pacientes com grau mais avançado da doença. Além disso, é difícil dizer se a procura pelo serviço médico é postergado até que a acuidade visual seja realmente muito ruim ou se esses pacientes têm maior intolerância às lentes de contato. Todavia, a 
acuidade visual corrigida foi semelhante entre os grupos e muito melhor com relação à acuidade visual pré-operatória (Tabela 3). Esses resultados, com corroboração na literatura $^{(28,67)}$, indicam que a condição refrativa pré-operatória não é crítica na predição dos resultados refrativos e visuais após transplante de córnea em olhos com ceratocone.

Levando-se em consideração todos os olhos da amostra (70 olhos), observou-se a AV média de 0,29 LogMAR (20/40) com a melhor correção com óculos (Tabela 3). No entanto, quando se remove da amostra os olhos com outras alterações, que poderiam interferir com os resultados dos transplantes de córnea, a AV média aumenta para 0,14 LogMAR (20/30), o que está de acordo com os dados da literatura (Tabela 8$)^{(2,7,16,27-28,30,62-63)}$.

A experiência do cirurgião, em alguns estudos, tem papel preponderante na sobrevida do enxerto ${ }^{(68)}$. Parece natural que cirurgiões que fizeram e fazem mais transplantes, tendem a ser mais diligentes em suas cirurgias.

Não foi encontrada diferença nas análises dos resultados visuais, refracionais e topográficos (Tabelas 3, 4, 5, 6, 7 e 8) entre os dois grupos. Esse resultado é corroborado na literatura por Gross et al. ${ }^{(69)}$, Kirkness et al. ${ }^{(16)}$ e Hommoudi et al. ${ }^{(70)}$, que não encontraram diferença nos resultados dos astigmatismos, assimetria e regularidade da superfície entre os transplantes realizados por cirurgiões experientes e estagiários (fellows) em serviço de treinamento em transplante de córnea ${ }^{(69-71)}$.

Teixeira et al. em seu estudo, de transplantes realizados por médicos em treinamento, quando se referem aos resultados nos transplantes de córnea por ceratocone, encontraram também resultados similares aos da literatura ${ }^{(72)}$, bem como os estudos conduzidos por Randleman et al., que analisam as indicações e os resultados de ceratoplastia penetrante realizada por cirurgiões residentes. No caso específico do ceratocone, tiveram um resultado de $87,5 \%$ de sobrevivência do enxerto com um seguimento pós-operatório médio de 21,9 meses, porém não houve a comparação dos resultados com cirurgiões experientes ${ }^{(73)}$. Provavelmente por não encontrarem diferença em seus resultados, outros autores que estudam os enxertos em serviços de córnea o fazem englobando os resultados dos transplantes de córnea realizados por cirurgiões experientes, bem como os realizados por seus estagiários ${ }^{(2,16,30)}$.

Similar a outros serviços de treinamento de cirurgiões em córnea $^{(52)}$, no Setor de Doenças Externas e Córnea da UNIFESP, todos os pacientes são examinados e aprovados para cirurgia pelo residente e por pelo menos um médico mais experiente denominado orientador. A cirurgia é realizada por residente do terceiro ano ou estagiário, com um ano a mais de experiência do que em outros centros de treinamento ${ }^{(52)}$, com a supervisão de um cirurgião mais experiente. Após a cirurgia, o pós-operatório é realizado no ambulatório do Serviço e os cirurgiões em treinamento são orientados no gerenciamento das medicações, manejo do astigmatismo e complicações pelos médicos orientadores.

Os resultados deste estudo estão em concordância com outros da literatura em que se compara a experiência dos cirurgiões, mas também difere destes, sendo que os resultados dos transplantes aqui analisados o foram após todas as suturas terem sido removidas e em um tempo de seguimento mais longo $(7,9 \text { anos })^{(69-72)}$.

\section{CONCLUSÃO}

A acuidade visual pré-operatória foi menor nos pacientes operados pelo grupo de cirurgiões em treinamento. Não obstante, houve melhora da acuidade visual em relação à préoperatória nos dois grupos e não foi preditiva da acuidade visual pós-operatória.

Houve correlação significativa do Grupo II, de cirurgiões em treinamento, com ocorrência de rejeição endotelial. As demais complicações foram semelhantes em ambos os grupos.

A experiência do cirurgião não teve influência nos resultados refracional, videoceratográfico e de acuidade visual após o transplante de córnea.

\section{AGRADECIMENTO}

Dr. Marcelo Cunha pelo apoio e disposição de pacientes que consentiram em participar deste estudo.

\section{ABSTRACT}

Purpose: Refractive and visual analysis in corneal transplantations performed for keratoconus by two groups of surgeons. Methods: Seventy eyes of seventy patients, which had been submitted to penetrating keratoplasty (PK) for keratoconus, were examined and their charts were reviewed retrospectively for long-term results after all sutures had been removed. The patients were divided into two groups, Group I had surgery performed by skillful surgeons and Group II by surgeons in training. The results of best correct visual acuity, refractive error, postoperative complications and computerized videokeratoscopy were compared. Results: The mean follow-up was 7.9 years with a range of $1.6-20$ years. Each group consisted of 35 eyes. There were 35 females and 35 males. The donor age was different between the two groups. The surgical technique was the same in both groups, but the number of sutures was different. Preoperative mean $\log$ MAR visual acuity was low in the two groups, but statistically different and no relationship was found between the results. All the refractive, topographic and visual acuity results were similar between both groups. Endothelial rejection was more frequent in Group II. Only three grafts lost graft clarity. These patients belonged to Group II. Conclusion: In a corneal referral practice, the visual results after PK were similar to those related to the PK performed by either expert surgeons or in training.

Keywords: Keratoconus; Corneal keratoplasty; Postoperative complications; Refractive error/astigmatism 


\section{REFERÊNCIAS}

1. Krachmer JH, Feder RS, Belin MW. Keratoconus and related noninflammatory corneal thinning disorders. Surv Ophthalmol. 1984;28(4):293-322.

2. Brierly SC, Izquierdo L Jr, Mannis MJ. Penetrating keratoplasty for keratoconus. Cornea. 2000;19(3):329-32.

3. Doyle SJ, Harper C, Marcyniuk B, Ridgway AE. Prediction of refractive outcome in penetrating keratoplasty for keratoconus. Cornea. 1996;15(5):441-5.

4. Polack FM. Lamellar keratoplasty. Malbran's "peeling off" technique. Arch Ophthalmol. 1971;86(3):293-5.

5. Wood TO. Lamellar transplants in keratoconus. Am J Ophthalmol. 1977;83 (4):543-5.

6. McDonnell PJ, Falcon MG. The lamellar corneal graft for optical indications. Eye. 1988;2(Pt 4):390-4

7. Watson SL, Ramsay A, Dart JK, Bunce C, Craig E. Comparison of deep lamellar keratoplasty and penetrating keratoplasty in patients with keratoconus. Ophthalmology. 2004;111(9):1676-82.

8. Kaufman HE, Werblin TP. Epikeratophakia for the treatment of keratoconus. Am J Ophthalmol. 1982;93(3):342-7.

9. McDonald MB, Safir A, Waring GO $3^{\text {rd }}$, Schlichtemeier WR, Kissling GE, Kaufman HE. A preliminary comparative study of epikeratophakia or penetrating keratoplasty for keratoconus. Am J Ophthalmol. 1987;103(3 Pt 2):467.

10. Nose W, Mattos RB, Belfort Jr R, Guedes M. Transplante de córnea e epiceratoplastia no ceratocone - Estudo prospectivo e comparativo. Arq Bras Oftalmol. 1989;52(4):135-6.

11. Wagoner MD, Smith SD, Rademaker WJ, Mahmood MA. Penetrating keratoplasty vs. epikeratoplasty for the surgical treatment of keratoconus. J Refract Surg. 2001;17(2):138-46.

12. Siganos D, Ferrara P, Chatzinikolas K, Bessis N, Papastergiou G. Ferrara intrastromal corneal rings for correction of keratoconus. J Cataract Refract Surg. 2002;28(11):1947-51.

13. Moreira H, Oliveira CS, Godoy G, Wahab SA. Anel intracorneano de Ferrara em ceratocone. Arq Bras Oftalmol. 2002;65(1):59-63.

14. Colin J, Velou S. Current surgical options for keratoconus. J Cataract Refract Surg. 2003;29(2):379-86. Review.

15. Miranda D, Sartori M, Francesconi C, Allemann N, Ferrara P, Campos M. Ferrara intrastromal corneal ring segments for severe keratoconus. J Refract Surg. 2003;19(6):645-53.

16. Kirkness CM, Ficker LA, Steele AD, Rice NS. The success of penetrating keratoplasty for keratoconus. Eye. 1990;4(Pt 5):673-88.

17. The collaborative corneal transplantation studies (CCTS). Effectiveness of histocompatibility matching in high-risk corneal transplantation. The Collaborative Corneal Transplantation Studies Research Group.Arch Ophthalmol. 1992;110(10):1392-403.

18. Laibson PR, Rapuano CJ. 100-year review of cornea. Ophthalmology. 1996;103(8 Suppl): S17-28.

19. Vail A, Gore SM, Bradley BA, Easty DL, Rogers CA, Armitate WJ. Clinical and surgical factors influencing corneal graft survival, visual acuity, and astigmatism. Corneal Transplant Follow-up Study Collaborators. Ophthalmology. 1996;103(1):41-9.

20. Thibos LN, Wheeler W, Horner D. Power vectors: an application of Fourier analysis to the description and statistical analysis of refractive error. Optom Vis Sci. 1997;74(6):367-75.

21. Karabatsas CH, Cook SD, Sparrow JM. Proposed classification for topographic patterns seen after penetrating keratoplasty. Br J Ophthalmol. 1999;83 (4):403-9.

22. Sharma V, Sharma N, Vajpayee RB, Titiyal JS, Sinha R. Study of corneal topographic patterns with single continuous suturing techniques in penetrating keratoplasty. Cornea. 2003;22(1):5-9.

23. Kennedy RH, Bourne WM, Dyer JA. A 48-year clinical and epidemiologic study of keratoconus. Am J Ophthalmol. 1986;101(3):267-73.

24. Lass JH, Lembach RG, Park SB, Hom DL, Fritz ME, Svilar M, et al. Clinical management of keratoconus. A multicenter analysis. Ophthalmology. 1990;97(4):433-45.

25. Rabinowitz YS. Keratoconus. Surv Ophthalmol. 1998;42(4):297-319. Review.

26. Price FW Jr, Whitson WE, Marks RG. Progression of visual acuity after penetrating keratoplasty. Ophthalmology. 1991;98(8):1117-85.

27. Koralewska-Makar A, Floren I, Stenevi U. The results of penetrating keratoplasty for keratoconus. Acta Ophthalmol Scand. 1996;74(2):187-90.

28. Lim L, Pesudovs K, Coster DJ. Penetrating keratoplasty for keratoconus: visual outcome and success. Ophthalmology. 2000;107(6):1125-31.
29. Brahma A, Ennis F, Harper R, Ridgway A, Tullo A. Visual function after penetrating keratoplasty for keratoconus: a prospective longitudinal evaluation. Br J Ophthalmol. 2000;84(1):60-6.

30. Sharif KW, Casey TA. Penetrating keratoplasty for keratoconus: complications and long-term success. Br J Ophthalmol. 1991;75(3):142-6.

31. Thalainen A. Clinical and epidemiological features of keratoconus genetic and external factors in the pathogenesis of the disease. Acta Ophthalmol Suppl. 1986;178:1-64.

32. Maeno A, Naor J, Lee HM, Hunter WS, Rootman DS. Three decades of corneal transplantation: indications and patient characteristics. Cornea. 2000; 19(1):7-11.

33. Swinger CA. Postoperative astigmatism. Surv Ophthalmol. 1987;31(4):219-48.

34. Duran JA, Malvar A, Diez E. Corneal dioptric power after penetrating keratoplasty. Br J Ophthalmol. 1989;73(8):657-60.

35. Solano JM, Hodge DO, Bourne WM. Keratometric astigmatism after suture removal in penetrating keratoplasty: double running versus single running suture techniques. Cornea. 2003;22(8):716-20.

36. Boisjoly HM, Tourigny R, Bazin R, Laughrea PA, Dube I, Chamberland G, et al. Risk factors of corneal graft failure. Ophthalmology. 1993;100(11):1728-35.

37. Price MO, Thompson RW Jr, Price FW Jr. Risk factors for various causes of failure in initial corneal grafts. Arch Ophthalmol. 2003;121(8):1087-92.

38. Khodadoust AA, Silverstein AM. Studies on the nature of the privilege enjoyed by corneal allografts. Invest Ophthalmol. 1972;11(3):137-48.

39. Waldock A, Cook SD. Corneal transplantation: how successful are we? Br J Ophthalmol. 2000;84(8):813-5.

40. Donshik PC, Cavanagh HD, Boruchoff SA, Dohlman CH. Effect of bilateral and unilateral grafts on the incidence of rejections in keratoconus. Am J Ophthalmol. 1979;87(6):823-6.

41. Vail A, Gore SM, Bradley BA, Easty DL, Rogers CA, Armitage WJ. Conclusions of the corneal transplant follow up study. Collaborating Surgeons. $\mathrm{Br}$ J Ophthalmol. 1997;81(8):631-6.

42. Maguire MG, Stark WJ, Gottsch JD, Stulting RD, Sugar A, Fink NE, Schwartz A. Risk factors for corneal graft failure and rejection in the collaborative corneal transplantation studies. Collaborative Corneal Transplantaion Studies Research Group. Ophthalmology. 1994;101(9):1536-47.

43. Williams KA, Roder D, Esterman A, Muehlberg SM, Coster DJ. Factors predictive of corneal graft survival. Report from the Australian Corneal Graft Registry. Ophthalmology. 1992;99(3):403-14.

44. Weisbrod DJ, Sit M, Naor J, Slomovic AR. Outcomes of repeat penetrating keratoplasty and risk factors for graft failure. Cornea. 2003;22(5):429-34.

45. Jonas JB, Rank RM, Budde WM. Immunologic graft reactions after allogenic penetrating keratoplasty. Am J Ophthalmol. 2002;133(4):437-43.

46. Tuft SJ, Gregory WM, Davison CR. Bilateral penetrating keratoplasty for keratoconus. Ophthalmology. 1995;102(3):462-8.

47. Volker-Dieben HJ, Kok-van Alphen CC, Lansbergen Q, Persijn GG. Different influences on corneal graft survival in 539 transplants. Acta Ophthalmol (Copenh). 1982;60(2): 190-202.

48. Epstein RJ, Seedor JA, Dreizen NG, Stulting RD, Warning GO $3^{\text {rd }}$, Wilson LA, Cavanagh HD. Penetrating keratoplasty for herpes simplex keratitis and keratoconus. Allograft rejection and survival. Ophthalmology. 1987;94(8):935-44.

49. Chalita MRC, Diazgranados EBM, Sato EH, Branco BC, Freitas D. Rejeição corneana pós transplante de córnea: análise de dados do Banco de Olhos do Hospital São Paulo - Escola Paulista de Medicina. Arq Bras Oftalmol. 2000;63(1):55-8.

50. Wood TO, West C, Kaufman HE. Control of intraocular pressure in penetrating keratoplasty. Am J Ophthalmol. 1972;74(4):724-8.

51. Cavalcanti MTD, Mahon M, Nóbrega DAT, Remígio MCA, Pires CS Ceratocone: resultados visuais, complicações e qualidade de vida após ceratoplastia penetrante realizada por médico residente. Arq Bras Oftalmol. 2004;67 (3):415-8.

52. Kutzscher EM, Sorenson AL, Goodman DF. Penetrating keratoplasty performed by residents. Arch Ophthalmol. 2004;122(9):1333-6.

53. Ehlers N, Olsen T. Long term results of corneal grafting in keratoconus. Acta Ophthalmol (Copenh). 1983;61(5):918-26.

54. Mader TH, Yuan R, Lynn MJ, Stulting RD, Wilson LA, Warning GO $3^{\text {rd }}$. Changes in keratometric astigmatism after suture removal more than one year after penetrating keratoplasty. Ophthalmology. 1993;100(1):119-26; discussion 127.

55. Williams KA, Ash JK, Pararajasegaram P, Harris S, Coster DJ. Long-term outcome after corneal transplantation. Visual result and patient perception of success. Ophthalmology. 1991;98(5):651-7.

56. Strelow S, Cohen EJ, Leavitt KG, Laibson PR. Corneal topography for selective suture removal after penetrating kerotoplasty. Am J Ophthalmol. 1991;112(6):657-65. 
57. Touzeau O, Borderie VM, Allouch C, Scheer S, Laroche L. Effects of penetrating keratoplasty suture removal on corneal topography and refraction. Cornea. $1999 ; 18(6): 638-44$

58. Jensen AD, Maumenee AE. Refractive error following keratoplasty. Trans Am Ophthalmol Soc. 1974;72:123-31

59. Troutman RC, Gaster RN. Surgical advances and results of keratoconus. Am J Ophthalmol. 1980;90(2):131-6.

60. De Lavalette JG, de Lavalette AR, van Rij G, Beekhuis WH, de BeijerDominicus JA. Long-term results of corneal transplantations in keratoconus patients. Doc Ophthalmol. 1985;59(1):93-7.

61. Spadea L, Bianco G, Mastrofini MC, Balestrazzi E. Penetrating keratoplasty with donor and recipient corneas of the same diameter. Ophthalmic Surg Lasers. 1996;27(6):452-30.

62. Buzard KA, Fundingsland BR. Corneal transplant for keratoconus: results in early and late disease. J Cataract Refract Surg. 1997;23(3):398-406.

63. Olson RJ, Pingree M, Ridges R, Lundergan ML, Alldredge C Jr, Clinch TE. Penetrating keratoplasty for keratoconus: a long-term review of results and complications. J Cataract Refract Surg. 2000;26(7):987-91.

64. Dolorico AM, Tayyani R, Ong HV, Gaster RN. Shortterm and longterm visual and astigmatic results of an opposing 10-0 nylon double running suture technique for penetrating keratoplasty. J Am Coll Surg. 2003;197(6):991-9.

65. Wilson SE, Klyce SD. Advances in the analysis of corneal topography. Surv Ophthalmol. 1991;35(4):269-77.
66. Khong AM, Mannis MJ, Plotnik RD, Johnson CA. Computerized topographic analysis of the healing graft after penetrating keratoplasty for keratoconus. Am J Ophthalmol. 1993;115(2):209-15.

67. Liu Y, Seitz B, Langenbucher A, Nguyen NX, Naumann GO. Impact of preoperative corneal curvature on the outcome of penetrating keratoplasty in keratoconus. Cornea. 2003;22(5):409-12.

68. Vail A, Gore SM, Bradley BA, Easty DL, Rogers CA. Corneal transplantation in the United Kingdom and Republic of Ireland. Br J Ophthalmol. 1993; 77(10):650-6.

69. Gross RH, Poulsen EJ, Davitt S, Schwab IR, Mannis MJ. Comparison of astigmatism after penetrating keratoplasty by experienced cornea surgeons and cornea fellows. Am J Ophthalmol. 1997;123(5):636-43.

70. Hammoudi DS, Segev F, Abdolell M, Rootman DS. Outcome of penetrating keratoplasty performed by cornea fellows compared with that of an experienced staff surgeon. Cornea. 2005;24(4):410-6.

71. Wiggins RE, Cobo M, Foulks GN. Results of penetrating keratoplasty by residents. Arch Ophthalmol. 1990;108(6):851-3.

72. Teixeira MF, Almeida Júnior GC, Rodrigues ML, Kamimoto PS, Kashiwabuchi LK. Resultados e indicações de ceratoplastias penetrantes realizadas por médicos em treinamento, num país em desenvolvimento. Arq Bras Oftalmol. 2001;64(6):557-61.

73. Randleman JB, Song CD, Palay DA. Indications for and outcomes of penetrating keratoplasty performed by resident surgeons. Am J Ophthalmol. 2003;136(1):68-75.

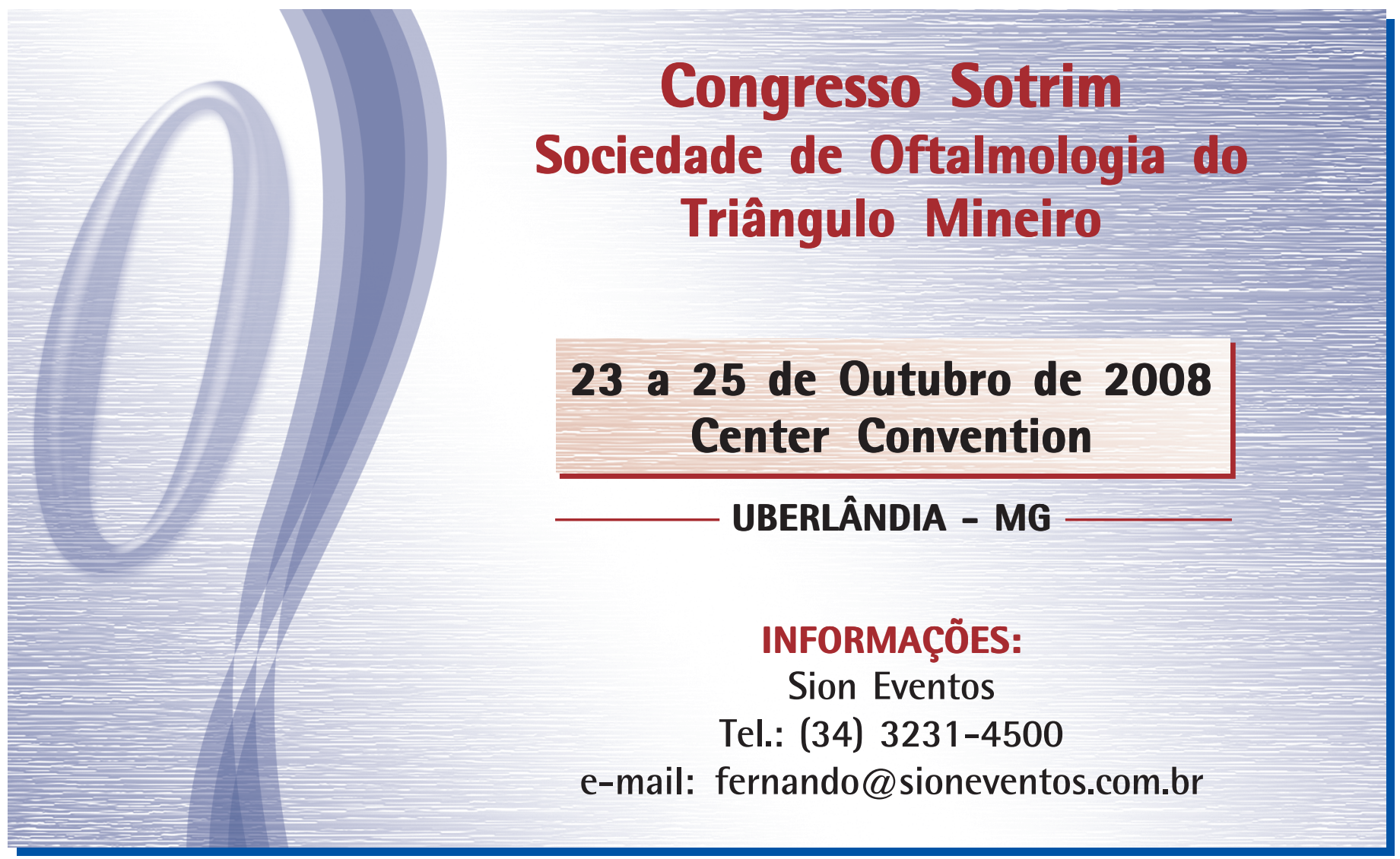

